\title{
REAL HYPERSURFACES AND COMPLEX SUBMANIFOLDS IN COMPLEX PROJECTIVE SPACE
}

\begin{abstract}
MAKOTO KIMURA
ABSTRACT. Let $M$ be a real hypersurface in $P^{\prime \prime}(\mathbf{C}), J$ be the complex structure and $\xi$ denote a unit normal vector field on $M$. We show that $M$ is (an open subset of) a homogeneous hypersurface if and only if $M$ has constant principal curvatures and $J \xi$ is principal. We also obtain a characterization of certain complex submanifolds in a complex projective space. Specifically, $P^{m}(\mathbf{C})$ (totally geodesic), $Q^{n}, P^{1}(\mathbf{C}) \times$ $P^{\prime \prime}(\mathrm{C}), S U(5) / S(U(2) \times U(3))$ and $S O(10) / U(5)$ are the only complex submanifolds whose principal curvatures are constant in the sense that they depend neither on the point of the submanifold nor on the normal vector.
\end{abstract}

0. Introduction. Many differential geometers have investigated isoparametric hypersurfaces in a sphere (i.e., submanifolds of codimension 1 with constant principal curvatures). In particular, Münzner [6, 7] showed that the number of distinct principal curvatures of isoparametric hypersurfaces in a sphere is $1,2,3,4$ or 6 . This result is not obtained by classfying all isoparametric hypersursfaces.

There are many papers about real hypersurfaces in complex projective space $P^{n}(\mathrm{C})$. In particular, Takagi [15] classfied all homogeneous real hypersurfaces in $P^{n}(\mathbf{C})$, and showed that the number of distinct principal curvatures of homogeneous real hypersurfaces in $P^{n}(\mathbf{C})$ is 2,3 or 5. Moreover, if a real hypersurface $M$ has 2 or 3 distinct constant principal curvatures, then $M$ is congruent to one of the homogeneous examples [16 and 17].

In Theorem 1, using the results of Münzner and Okumura, we show that if a real hypersurface $M$ of $P^{n}(\mathbf{C})$ has constant principal curvatures and if the tangent vector field $J \xi$, which is obtained by applying the complex structure $J$ to the unit normal vector field $\xi$, is principal, then the number of distinct principal curvatures of $M$ is 2,3 or 5 .

Cecil and Ryan [4] studied real hypersurfaces in $P^{n}(\mathbf{C})$ on which $J \xi$ is principal. In fact, the tangent vector field $J \xi$ on a tube over a Kähler submanifold is principal. Conversely, if $J \xi$ is principal on a real hypersurface and the rank of focal map is constant (\$2), then $M$ lies on a tube over a Kähler submanifold. Making use of these these results, we have

THEOREM 4. Let $M$ be a connected real hypersurface in $P^{\prime \prime}(\mathbf{C})$. Then $M$ has constant principal curvatures and $J \xi$ is principal if and only if $M$ is congruent to an open subset of a homogeneous real hypersurface.

Received by the editors October 30, 1984 and, in revised form, July 8, 1985.

1980 Mathematics Subject Classification. Primary 53B25, 53C40.

Key words and phrases. Real hypersurfaces, complex submanifolds, focal sets, tubes, shape operators. 
At the same time, we get the following result concerning Kähler submanifolds.

THEOREM 5. Let $N$ be a Kähler submanifold of a complex projective space. Then eigenvalues of the shape operator $A_{\xi}$ are constant in the sense that they depend neither on the point $x$ in $N$ nor on the unit normal vector $\xi$ if and only if $N$ is congruent to an open subset of one of the following:

$$
\begin{aligned}
& P^{n} \rightarrow P^{m}(\text { totally geodesic }), \\
& Q^{n} \rightarrow P^{n+1}(\text { complex quadric }) \\
& P^{1} \times P^{n-1} \rightarrow P^{2 n-1}, \\
& S U(5) / S(U(3) \times U(2)) \rightarrow P^{9}, \\
& S O(10) / U(5) \rightarrow P^{15} .
\end{aligned}
$$

In the last section, we get some results about noncomplex focal manifolds of homogeneous real hypersurfaces in $P^{n}(\mathbf{C})$.

The author would like to express his thanks to Professor K. Ogiue, Mr. N. Ejiri, Mr. Y. Shimizu and Mr. S. Udagawa for valuable comments.

1. Preliminaries. First of all, we recall the Fubini-Study metric on the complex projective space $P^{n}(\mathbf{C})$ (or $P^{n}$ ). For a more detailed description, see [4]. Let $(z, w)=\sum_{k=0}^{n} z^{k} \bar{w}^{k}$ be the natural Hermitian inner product on $\mathbf{C}^{n+1}$. The Euclidean metric $\langle$,$\rangle on \mathbf{C}^{n+1}$ is given by $\langle z, w\rangle=\operatorname{Re}(z, w)$. The unit sphere in $\mathbf{C}^{n+1}$ is the principal fibre bundle over $P^{n}(\mathbf{C})$ with the structure group $S^{1}$ and the projection map $\pi$. The tangent space of $S^{2 n+1}$ at a point $z$ is

$$
T_{z} S^{2 n+1}=\left\{w \in \mathbf{C}^{n+1} \mid\langle z, w\rangle=0\right\} .
$$

Let

$$
T_{z}^{\prime}=\left\{w \in \mathbf{C}^{n+1} \mid\langle z, w\rangle=\langle i z, w\rangle=0\right\} .
$$

The distribution $T_{z}^{\prime}$ defines a connection in the principal fibre bundle $S^{2 n+1}\left(P^{n}(\mathbf{C}), S^{1}\right)$, because $T_{z}^{\prime}$ is complementary to the subspace $\{i z\}$ tangent to the fibre through $z$, and invariant under the $S^{1}$-action. Then the Fubini-Study metric $g$ of constant holomorphic sectional curvature $c$ is given by $g(X, Y)=(c / 4)\left\langle X^{*}, Y^{*}\right\rangle$, where $X, Y \in T_{x} P^{n}(\mathbf{C})$, and $X^{*}, Y^{*}$ are respectively their horizontal lifts at a point $z$ with $\pi(z)=x$. In this paper, we set $c=4$, unless otherwise stated. The complex structure on $T^{\prime}$ defined by multiplication by $\sqrt{-1}$ induces a canonical complex structure on $P^{n}(\mathrm{C})$ through $\pi_{*}$.

2. Focal sets and tubes in $P^{n}(\mathbf{C})$. In this section, we review results about focal sets and tubes in $P^{n}(\mathbf{C})$, obtained by Cecil and Ryan. Let $M$ be an embedded real $n$-dimensional $C^{\infty}$-submanifold of $P^{m}(\mathbf{C})$ with $m=(n+p) / 2$. We denote by $N M$ the normal bundle over $M$ with projection $P$ onto $M$. For $\xi \in N M$, let $F(\xi)$ be the point in $P^{m}(\mathbf{C})$ reached by transversing a distance $|\xi|$ along the geodesic in $P^{m}(\mathbf{C})$ originating at $x=P(\xi)$ with initial tangent vector $\xi$. A point $y \in P^{m}(\mathbf{C})$ is called a focal point of multiplicity $\nu>0$ of $(M, x)$ if $y=F(\xi)$ for some $\xi$ and the Jacobian of $F$ has nullity $\nu$ at $\xi$. 
Let $\left\{\xi_{1}, \ldots, \xi_{p}\right\}$ be an orthonormal basis for the normal space $T_{x}{ }^{\perp} M$ with $\xi_{1}=\xi$. Let $U$ be a sufficiently small normal coordinate neighborhood of $x$. Extend $\xi_{1}, \ldots, \xi_{p}$ to orthonormal normal vector fields on $U$ by parallel translation with respect to the normal connection $\nabla^{\perp}$ along geodesics through $x$ in $M$. For any $u \in U$ and $\eta \in T_{u}^{\perp} M$, we can write

$$
\eta=\mu\left(\left(1-\sum_{j=2}^{p} t_{j}^{2}\right)^{1 / 2} \xi_{1}+t_{2} \xi_{2}+\cdots+t_{p} \xi_{p}\right)
$$

where $0 \leqslant \mu, 0 \leqslant\left|t_{j}\right| \leqslant 1$ for all $j$ and $\sum_{j=2}^{p} t_{j}^{2} \leqslant 1$. Note that $\mu=|\eta|$ and the $t_{j}$ are the direction cosines of $\eta$. The tangent space at this point $\eta$ in $N M$ can be considered as

$$
T_{u} M \times \operatorname{span}\left\{\partial / \partial \mu, \partial / \partial t_{2}, \ldots, \partial / \partial t_{p}\right\}
$$

Cecil and Ryan studied the focal points of $M$ in the case where $M$ is a complex submanifold or $J \xi$ is a principal vector of $A_{\xi}$. Their result can be stated as follows. Here $T_{\lambda}$ denotes the eigenspace corresponding to an eigenvalue $\lambda$ of $A_{\xi}$. As in [4], we assume that $0<r \leqslant \pi / 2$.

Proposition 2.1 [4]. Suppose $\xi_{2}=J \xi$ is normal to $M$ at $x$. Then

(a) $\left(F_{*}\right)_{r \xi}(X, 0)=0$ if $\lambda=\cot r$ is an eigenvalue of $A_{\xi}$ and $X \in T_{\lambda}$,

(b) $\left(F_{*}\right)_{r \xi}\left(\partial / \partial t_{2}\right)=0$ for $r=\pi / 2$,

(c) $\left(F_{*}\right)_{r \xi}(X, V) \neq 0$ except as determined by (a) and (b).

Proposition 2.2 [4]. Suppose J $\xi$ is an eigenvector of $A_{\xi}$ with corresponding eigenvalue $\mu$. Then

(a) $\left(F_{*}\right)_{r \xi}(X, 0)=0$ if $\lambda=\cot r$ is an eigenvalue of $A_{\xi}$ and $X$ is a vector in $T_{\lambda}$ orthogonal to $J \xi$.

(b) $\left(F_{*}\right)_{r \xi}(J \xi, 0)=0$ if $\mu=2 \cot 2 r$,

(c) $\left(F_{*}\right)_{r \xi}(X, V) \neq 0$ except as determined by (a) and (b).

Next, we review the computation of the shape operator of a tube over some special submanifold $M$ of $P^{m}(\mathrm{C})$. Let $B M$ denote the bundle of unit normal vectors to $M$. The tube of radius $r$ over $M$ is defined by the map $\phi_{r}$ of $B M$ into $P^{m}(\mathbf{C})$ given by $\phi_{r}(\xi)=F(r \xi)$. For sufficiently small values of $r$, at least, $\phi_{r}$ determines a real hypersurface of $P^{m}(\mathbf{C})$. In the special case where $M$ is a real hypersurface, it is customary to consider $\phi_{r}$ as a map of $M$ into $P^{m}(\mathbf{C})$. If $\xi$ is a local field of unit normals, then $\phi_{r}(x)=F(r \xi(x))$. For values of $r$ such that $\phi_{r}$ is an immersion, $\phi_{r} M$ is called the parallel hypersurface at oriented distance $r$ from $M$.

Let $A_{r}$ be the shape operator of the tube $\phi_{r}$ at the point $\xi \in B M$. Using the same trivialization of $N M$ as before, we see that $T_{\xi} B M$ is isomorphic to $T_{x} M \times$ $\operatorname{span}\left\{\partial / \partial t_{2}, \ldots, \partial / \partial t_{p}\right\}$.

Proposition 2.3 [4]. Suppose $\xi_{2}=J \xi$ is normal to $M$ at $x$. Let $X_{1}, \ldots, X_{n}$ be a basis of principal vectors of $A_{\xi}$ with corresponding principal curvatures $\lambda_{j}=\cot \theta_{j}$, 
$0<\theta_{j}<\pi$. Then the shape operator $A_{r}$ of the tube $\phi_{r}$ is given in terms of its principal vectors by

(a) $A_{r}\left(\partial / \partial t_{2}\right)=-2 \cot 2 r\left(\partial / \partial t_{2}\right)$,

(b) $A_{r}\left(\partial / \partial t_{j}\right)=-\cot r\left(\partial / \partial t_{j}\right), 3 \leqslant j \leqslant p$,

(c) $A_{r}\left(X_{j}, 0\right)=\cot \left(\theta_{j}-r\right)\left(X_{j}, 0\right), 1 \leqslant j \leqslant n$.

Proposition 2.4 [4]. Suppose $J \xi$ is an eigenvector of $A_{\xi}$ with corresponding eigenvalue $2 \cot 2 \theta, 0<\theta<\pi / 2$. Suppose $J \xi, X_{2}, \ldots, X_{n}$ is a basis of principal vectors of $A_{\xi}$ with $A_{\xi} X_{j}=\cot \theta_{j} X_{j}, 2 \leqslant j \leqslant n, 0<\theta_{j}<\pi$. Then the shape operator $A_{r}$ of the tube $\phi_{r}$ is given in terms of its principal vectors by

(a) $A_{r}\left(\partial / \partial t_{j}\right)=-\cot r\left(\partial / \partial t_{j}\right), 2 \leqslant j \leqslant p$,

(b) $A_{r}\left(X_{j}, 0\right)=\cot \left(\theta_{j}-r\right)\left(X_{j}, 0\right), 2 \leqslant j \leqslant n$,

(c) $A_{r}(J \xi, 0)=2 \cot (2(\theta-r))(J \xi, 0)$.

Using Proposition 2.3, we have the following result:

COROLlaRY 2.5 [4]. Let $M$ be a real hypersurface in $P^{m}(\mathbf{C})$ which lies on a tube of constant radius over a complex submanifold of $P^{m}(\mathbf{C})$. Let $\xi$ be a unit normal vector to $M$. Then $J \xi$ is a principal vector of the shape operator $A_{\xi}$.

Corollary 2.5 shows that there are many real hypersurfaces with $J \xi$ principal and the condition that $J \xi$ is principal is natural to a certain extent. Conversely, we show next that with certain restrictions, if $J \xi$ is principal, then the real hypersurface must lie on a tube of constant radius over a complex submanifold of $P^{m}(\mathbf{C})$.

REMARK 2.6. Maeda [5] proved that if $J \xi$ is principal, then the corresponding principal curvature $\mu$ is locally constant.

Proposition 2.7 [4]. Let $M$ be a connected, orientable real hypersurface of $P^{m}(\mathbf{C})$ on which $J \xi$ is a principal vector with corresponding constant principal curvature $\mu=2 \cot 2 r$. Suppose the map $\phi_{r}$ has constant rank $q$. Then $q$ is even and every point $x_{0} \in M$ has a neighborhood $U$ such that $\phi_{r} U$ is an embedded complex (q/2)-dimensional submanifold of $P^{m}(\mathbf{C})$. Moreover, if $T_{0}$ is the foliation defined by integrable distribution $T_{0}(x)=\left\{X \in T_{x} M \mid\left(\phi_{r}\right)_{*} X=0\right\}$ for each $x \in U$, then the leaf of the foliation $T_{0}$ through $x$ intersects $U$ in an open subset of a geodesic hypersphere in the totally geodesic $P^{m-(q / 2)}(\mathbf{C})$ orthogonal to $T_{y}\left(\phi_{r} U\right)$ at $y=\phi_{r}(x)$. Thus $U$ lies on the tube of radius $r$ over $\phi_{r} U$.

3. Real hypersurfaces in a complex projective space. Suppose that $M$ is a real hypersurface in a complex projective space, and that the principal curvatures on $M$ are constant. We begin this section by showing (Theorem 1) that the number of distinct principal curvatures is 2,3 or 5 if $J \xi$ is principal.

Let $M^{2 n-1}$ and $\bar{M}^{2 n}$ be (real) hypersurfaces of $P^{n}(\mathbf{C})$ and $S^{2 n+1}$, respectively, such that the following diagram is commutative:

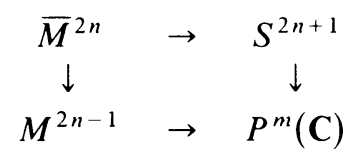


Assume that $\xi$ is the unit normal vector field on $M$ such that the tangent vector $J \xi$ is principal. Let $J \xi, e_{2}, \ldots, e_{2 n-1}$ be an orthonormal basis of principal vectors with principal curvatures $\mu, \lambda_{2}, \ldots, \lambda_{2 n-1}$, and $V$ be the vertical vector of the above submersion. Identify vector fields on $M$ and $P^{n}(\mathbf{C})$ with their horizontal lifts, and regard $V, J \xi, e_{2}, \ldots, e_{2 n-1}$ as the lift of the basis $J \xi, e_{2}, \ldots, e_{2 n-1}$. If $A$ (resp. $\bar{A}$ ) is the shape operator of the immersion $M \rightarrow P^{n}(\mathbf{C})$ (resp. $\bar{M} \rightarrow S^{2 n+1}$ ), then the relation between $A$ and $\bar{A}$ is given as follows.

Proposition 3.1 [11]. The matrix of $\bar{A}$ with respect to the above basis of the tangent space of $\bar{M}$ has the form

$$
\left(\begin{array}{ccccc}
0 & 1 & 0 & \cdots & 0 \\
1 & \mu & 0 & \cdots & 0 \\
0 & 0 & \lambda_{2} & \cdots & 0 \\
\vdots & \vdots & \vdots & \ddots & \vdots \\
0 & 0 & 0 & \cdots & \lambda_{2 n-1}
\end{array}\right)
$$

where the $\lambda_{1}, \ldots, \lambda_{2 n-1}$ are not necessarily distinct.

For isoparametric hypersurfaces in a sphere, Münzner obtained the following result in which the values $\bar{\lambda}_{1}, \ldots, \bar{\lambda}_{\bar{d}}$ must be distinct.

Proposition $3.2[1,6,7]$. Let $\bar{\lambda}_{1}, \ldots, \bar{\lambda}_{\bar{d}}$, be the distinct principal curvatures of an isoparametric hypersurface in a unit sphere and $\bar{m}_{1}, \ldots, \bar{m}_{\bar{d}}$, be their multiplicities. Then

(a) $\bar{d}=1,2,3,4$ or 6 ,

(b) $\bar{m}_{1}=\bar{m}_{2}=\bar{m}_{3}$ if $\bar{d}=3 ; \bar{m}_{1}=\bar{m}_{3}$ and $\bar{m}_{2}=\bar{m}_{4}$ if $\bar{d}=4$; and $\bar{m}_{1}=\bar{m}_{2}=$ $\cdots=\bar{m}_{6}=1$ or 2 if $\bar{d}=6$,

(c) there is a number $r$ such that $0<r<\pi / \bar{d}$ and

$$
\bar{\lambda}_{k}=\cot ((k \pi / \bar{d})-r) \quad(k=1, \ldots, \bar{d}),
$$

where $r$ is given as the distance from some focal set in $S^{2 n+1}$.

Using the above result, we obtain the following theorem.

THEOREM 1. Let $M$ be a real hypersurface of $P^{n}(\mathbf{C})$ and $\xi$ be a unit normal vector field on $M$. Suppose $M$ has distinct constant principal curvatures and $J \xi$ is principal. Then $d=2,3$ or 5 .

Proof. From the assumption and Proposition 3.1, $\bar{M}$ is an isoparametric hypersurface of $S^{2 n+1}$, and we can see that $\bar{M}$ has principal curvatures

$$
\left(\mu \pm\left(\mu^{2}+4\right)^{1 / 2}\right) / 2, \lambda_{2}, \ldots, \lambda_{2 n-1} .
$$

If we put $\mu=-2 \cot 2 r$ for $0<r<\pi / 2$, then

$$
\left(\mu+\left(\mu^{2}+4\right)^{1 / 2}\right) / 2=\tan r(=\cot (\pi / 2-r))
$$


and

$$
\left(\mu-\left(\mu^{2}+4\right)^{1 / 2}\right) / 2=-\cot r .
$$

Hence, using Proposition 3.2, we see that $\bar{d}$ (number of distinct constant principal curvatures of $\bar{M}$ ) is not equal to 1 or 3 . Next, we show that $\bar{d} \neq 6$.

We suppose that $\bar{M}$ has 6 distinct constant principal curvatures. From Proposition 3.2 , we can write $\bar{\lambda}_{k}=\cot ((k-1) \pi / 6-r)$ for $k=1, \ldots, 6$, and we have $\bar{m}_{1}=\bar{m}_{2}=\cdots=\bar{m}_{6}$. Let $\bar{m}=\bar{m}_{1}$. Then, making use of Proposition 3.1, we see that the principal curvatures of $M$ are given as follows:

$\lambda_{1}=-\cot r$ if $\bar{m} \neq 1$,

$\lambda_{4}=\cot (\pi / 2-r)$ if $\bar{m} \neq 1$,

$\lambda_{k}=\cot ((k-1) \pi / 6-r), k=2,3,5,6$,

$\mu=-2 \cot 2 r$.

Now, we consider the focal manifold $\tilde{M}=M_{\pi / 6}$ corresponding to

$$
\lambda_{2}=\cot (\pi / 6-r)
$$

in Proposition 2.2(a). Let $\bar{r}=\pi / 6-r, \xi$ be a unit normal to $M$ at the point $x$, and $\eta(x)=\pi\left(-\sin \bar{r} w+\cos \bar{r} \xi^{\prime}\right)$, where $w \in S^{2 n+1}$ with $\pi(w)=x$ and $\xi^{\prime}$ is the horizontal lift of $\xi$ to $T_{w}^{\prime}$. From the proof of Theorem 1 in [4], $\eta(x)$ is a unit normal vector of $\tilde{M}$ at $\bar{x}=\phi_{\bar{r}}(x)$ and $\eta$ gives a local diffeomorphism from $C=\left(\phi_{\bar{r}}\right)^{-1}(\bar{x})$ to $B_{\bar{x}} \tilde{M}$, where $B_{\bar{x}} \tilde{M}$ is the unit normal space of $\tilde{M}$ at $\bar{x}$. Hence $\eta(C)$ is open and, moreover, eigenvalues of the shape operator $A_{\eta(x)}$ of $\tilde{M}$ are:

$$
\begin{aligned}
& \cot 5 \pi / 6=-\sqrt{3} \text { if } \bar{m} \neq 1, \\
& \cot \pi / 3=1 / \sqrt{3} \text { if } \bar{m} \neq 1, \\
& \cot \pi / 6=\sqrt{3}, \cot \pi / 2=0, \cot 2 \pi / 3=-1 \sqrt{3}, \\
& -2 \cot \pi / 3=-2 / \sqrt{3} .
\end{aligned}
$$

Now let $t \in \mathbf{R}, \eta$ be a unit normal vector on $\tilde{M}$ and $\chi_{t}(\zeta)=\operatorname{det}\left(A_{\zeta}-t I\right)$ be the characteristic polynomial of $A_{\zeta}$. From above, $\left.\chi_{t}\right|_{\eta(C)}$ is constant. Since $\left.\chi_{t}\right|_{B_{v} \tilde{M}}$ is analytic and $\eta(C)$ is open in $B_{\bar{x}} \tilde{M}, \chi_{t}$ is constant on $B_{\bar{x}} \tilde{M}$. Hence eigenvalues of $A_{\eta}$ must not depend on the unit normal vector $\eta$ to $\tilde{M}$. In particular, the eigenvalues of $A_{\eta}$ coincide with the eigenvalues of $A_{-\eta}$. Hence, if $\lambda$ is a nonzero eigenvalue of $A_{\eta}$, then so is $-\lambda$. This contradicts the above fact. Consequently, we have $\bar{d}=2$ or 4 .

From Proposition 3.2, principal curvatures of $\bar{M}$ are written as:

Case $\bar{d}=2: \bar{\lambda}_{1}=-\cot r, m\left(\bar{\lambda}_{1}\right)=\bar{m}_{1}$,

$$
\bar{\lambda}_{2}=\cot (\pi / 2-r), m\left(\bar{\lambda}_{2}\right)=\bar{m}_{2}
$$

Case $\bar{d}=4: \bar{\lambda}_{1}=\cot r, m\left(\bar{\lambda}_{1}\right)=\bar{m}_{1}$,

$$
\begin{aligned}
& \bar{\lambda}_{2}=\cot (\pi / 4-r), m\left(\bar{\lambda}_{2}\right)=\bar{m}_{2}, \\
& \bar{\lambda}_{3}=\cot (\pi / 2-r), m\left(\bar{\lambda}_{3}\right)=\bar{m}_{1}, \\
& \bar{\lambda}_{4}=\cot (3 \pi / 4-r), m\left(\bar{\lambda}_{4}\right)=\bar{m}_{2},
\end{aligned}
$$

where $m(\lambda)$ denotes the multiplicity of the principal curvature $\lambda$. Then principal curvatures of $M$ are given as follows:

Case $\bar{d}=2$. If $\bar{m}_{1}=1$ or $\bar{m}_{2}=1$, then $d=2, \mu=-2 \cot 2 r$ and $\lambda=-\cot r$ or $\cot (\pi / 2-r)$. If $\bar{m}_{1}>1$ and $\bar{m}_{2}>1$, then $d=3$ and $\mu=-2 \cot 2 r, \lambda_{1}=-\cot r$, $\lambda_{2}=\cot (\pi / 2-r)$. 
Case $\bar{d}=4$. If $\bar{m}_{1}=1$, then $d=3$ and $\lambda_{1}=\cot (\pi / 4-r), m\left(\lambda_{1}\right)=n-1$, $\lambda_{2}=\cot (3 \pi / 4-r), m\left(\lambda_{2}\right)=n-1, \mu=-2 \cot 2 r, m(\mu)=1$. If $\bar{m}_{1}>1$, then $d=5$ and $\lambda_{1}=-\cot r, m\left(\lambda_{1}\right)=\bar{m}_{1}-1, \lambda_{2}=\cot (\pi / 4-r), m\left(\lambda_{2}\right)=\bar{m}_{2}, \lambda_{3}=$ $\cot (\pi / 2-r), m\left(\lambda_{3}\right)=\bar{m}_{1}-1, \lambda_{4}=\cot (3 \pi / 4-r), m\left(\lambda_{4}\right)=\bar{m}_{2}, \mu=-2 \cot 2 r$, $m(\mu)=1$.

Homogeneous real hypersurfaces of $P^{n}(\mathbf{C})$ are determined by Takagi [15]. On the other hand, there are several results that characterize homogeneous examples with two or three distinct principal curvatures among real hypersurfaces in $P^{n}(\mathbf{C})$. For example, we have the following two propositions.

Proposition 3.3 [4]. Let $M$ be a connected real hypersurface in $P^{n}(\mathbf{C})(n \geqslant 3)$ with at most two distinct principal curvatures at each point. Then $M$ is an open subset of a geodesic hypersphere.

Proposition 3.4 (DUE TO CECIL-RYAN). Let $M$ be a connected real hypersurface in $P^{\prime \prime}(\mathbf{C}), n \geqslant 3$, on which $J \xi$ is principal, which has three distinct principal curvatures at each point. Then $M$ is an open subset of a tube over a totally geodesic $P^{k}(\mathbf{C})$, $0<k<m-1$, or a tube over a complex quadric $Q^{m-1}$.

The proof is exactly the same as that of Theorem 4 of [4]. In the case $d=5$, we get the following result.

THEOREM 2. Let $M$ be a connected real hypersurface in $P^{n}(\mathbf{C})$ with five distinct constant principal curvatures on which $J \xi$ is principal. Then $M$ is congruent to an open subset of some homogeneous real hypersurface with $d=5$.

This theorem is related to the following theorem about the Kähler submanifold in a complex projective space.

THEOREM 3. Let $N$ be a connected n-dimensional Kähler submanifold of $P^{n+p}(\mathbf{C})$ such that eigenvalues of $A_{\xi}$ are $\lambda,-\lambda$ and 0 , where $\lambda$ is constant in the sense that it depends neither on the point $x$ in $N$ nor on the unit normal vector $\xi$. Then $N$ is congruent to an open subset of $P^{1} \times P^{n-1}$ with $n \geqslant 3$ or of $S U(5) / S(U(3) \times U(2))$ or $S O(10) / U(5)$.

REMARK 3.5. In Theorem 2, if the principal curvature in the direction of $J \xi$ is $2 \cot 2 r$, then the focal manifold $\phi_{r}(M)$ satisfies the assumption of Theorem 3. Conversely, in Theorem 3, the tube over $N$ satisfies the assumption of Theorem 2. Hence Theorem 2 is equivalent to Theorem 3. Moreover, making use of the above argument and Proposition 3.2, we see that principal curvatures of $M$ in Theorem 2 are given by $\lambda_{1}=-\cot r, \quad \lambda_{2}=\cot (\pi / 4-r), \quad \lambda_{3}=\cot (\pi / 2-r), \quad \lambda_{4}=$ $\cot (3 \pi / 4-r)$, and $\mu=-2 \cot 2 r$ with $m\left(\lambda_{1}\right)=m_{1}, m\left(\lambda_{2}\right)=m_{2}, m\left(\lambda_{3}\right)=m_{1}$, $m\left(\lambda_{4}\right)=m_{2}, m(\mu)=1$ and $m_{1}+m_{2}=n-1$. Finally, using Propositions 2.2 and 2.7, we find that $\lambda$ in Theorem 3 is equal to $1, m_{1}$ is even and $2 p=m_{1}+2$.

4. Kähler submanifolds in a complex projective space. Let $N$ be an $n$-dimension Kähler submanifold in $P^{n+p}(\mathbf{C})$ such that, for any unit normal vector field $\xi$ on $N$, eigenvalues of $A_{\xi}$ are $1,-1,0$ with $m(1)=m(-1)=m$ and $m(0)=2 n-2 m$. Then 
$A_{\xi}^{3}=A_{\xi}$. We now define an inner product $\langle$,$\rangle on T_{x}{ }^{\perp} N$ introduced by Cecil [3]. Let $\xi$ and $\eta$ be normal vectors at a point $x$ in $N$. If

$$
\langle\xi, \eta\rangle=\operatorname{trace} A_{\xi} A_{\eta},
$$

then $\langle$,$\rangle is an inner product on T_{x}{ }^{\perp} N$ which satisfies

$$
\langle J \xi, J \eta\rangle=\langle\xi, \eta\rangle, \quad\langle\xi, J \xi\rangle=0 .
$$

Let $\|\cdot\|$ denote the norm induced from the standard inner product $g$. If we fix a normal vector field $\xi$ to $N$ with $\|\xi\|=1$, then taking a suitable orthonormal basis of the tangent space of $N$, we see that

$$
A_{\xi}=\left(\begin{array}{ccc}
I_{m} & 0 & 0 \\
0 & -I_{m} & 0 \\
0 & 0 & 0
\end{array}\right), \quad A_{J \xi}=\left(\begin{array}{ccc}
0 & I_{m} & 0 \\
I_{m} & 0 & 0 \\
0 & 0 & 0
\end{array}\right),
$$

where $I_{m}$ denotes the $(m \times m)$ identity matrix. We also fix such a basis of the tangent space of $N$.

LEMMA 4.1. If $\eta \in T_{x}^{\perp} N$ with $\|\eta\|=1$ and $\langle\eta, \xi\rangle=\langle\eta, J \xi\rangle=0$, then

$$
A_{\eta}=\left(\begin{array}{ccc}
0 & 0 & { }^{t} C_{\eta} \\
0 & 0 & { }^{t} C_{\eta} J^{\prime} \\
C_{\eta} & -J^{\prime} C_{\eta} & 0
\end{array}\right),
$$

where $C_{\eta}$ is a $((2 n-2 m) \times m)$ matrix, and

$$
J^{\prime}=\left(\begin{array}{cc}
0 & -I_{n-m} \\
I_{n-m} & 0
\end{array}\right)
$$

Proof. We put

$$
\begin{gathered}
T^{+}=\left\{X \in T_{x} N \mid A_{\xi} X=X\right\}, \quad T^{-}=\left\{X \in T_{x} N \mid A_{\xi} X=-X\right\}, \\
T^{0}=\left\{X \in T_{x} N \mid A_{\xi} X=0\right\} .
\end{gathered}
$$

For $X \in T^{0}$, set $A_{\eta} X=Y_{+}+Y_{-}+Y_{0}$, where $Y_{+} \in T^{+}, Y_{-} \in T^{-}$and $Y_{0} \in T^{0}$. First, we will show that $Y_{0}=0$. Here

$$
A_{\xi+\eta}^{3} X=\|\xi+\eta\|^{2} A_{\xi+\eta} X=\|\xi+\eta\|^{2}\left(Y_{+}+Y_{-}+Y_{0}\right) .
$$

On the other hand, since $A_{\xi} X=0$,

$$
\begin{aligned}
A_{\xi+\eta}^{3} X & =\left(A_{\xi}^{2} A_{\eta}+A_{\xi} A_{\eta}^{2}+A_{\eta} A_{\xi} A_{\eta}+A_{\eta}^{3}\right) X \\
& =2 Y_{+}+2 Y_{-}+Y_{0}+\left(A_{\xi} A_{\eta}+A_{\eta} A_{\xi}\right) A_{\eta} X .
\end{aligned}
$$

Similarly,

$$
A_{\xi-\eta}^{3} X=\|\xi-\eta\|^{2} A_{\xi-\eta} X=-\|\xi-\eta\|^{2}\left(Y_{+}+Y_{-}+Y_{0}\right)
$$

and

$$
\begin{aligned}
A_{\xi-\eta}^{3} X & =\left(-A_{\xi}^{2} A_{\eta}+A_{\xi} A_{\eta}^{2}+A_{\eta} A_{\xi} A_{\eta}-A_{\eta}^{3}\right) \\
& =-\left(2 Y_{+}=2 Y_{-}+Y_{0}\right)+\left(A_{\xi} A_{\eta}+A_{\eta} A_{\xi}\right) A_{\eta} X
\end{aligned}
$$


Hence,

$$
\begin{aligned}
A_{\xi+\eta}^{3} X-A_{\xi-\eta}^{3} X & =\left(\|\xi+\eta\|^{2}+\|\xi-\eta\|^{2}\right)\left(Y_{+}+Y_{-}+Y_{0}\right) \\
& =4 Y_{+}+4 Y_{-}+2 Y_{0}
\end{aligned}
$$

Since $\|\xi+\eta\|^{2}+\|\xi-\eta\|^{2}=4$, we get $Y_{0}=0$.

Next, for $X \in T^{+}$, put $A_{\eta} X=Y_{+}+Y_{-}+Y_{0}$, where $Y_{+} \in T^{+}, Y_{-} \in T^{-}$and $Y_{0} \in$ $T^{0}$. We will show that $Y_{+}=($const $) \cdot X$. Here

$$
A_{\xi+\eta}^{3} X=\|\xi+\eta\|^{2} A_{\xi+\eta} X=\|\xi+\eta\|^{2}\left(X+Y_{+}+Y_{-}+Y_{0}\right)
$$

and

$$
A_{\xi+\eta}^{3} X=\left(A_{\xi}^{3}+A_{\xi}^{2} A_{\eta}+A_{\xi} A_{\eta} A_{\xi}+A_{\xi} A_{\eta}^{2}+A_{\eta} A_{\xi}^{2}+A_{\eta} A_{\xi} A_{\eta}+A_{\eta}^{2} A_{\xi}+A_{\eta}^{3}\right) X .
$$

Similarly,

$$
A_{\xi-\eta}^{3} X=\|\xi-\eta\|^{2} A_{\xi-\eta} X=\|\xi-\eta\|^{2}\left(X-Y_{+}-Y_{-}-Y_{0}\right)
$$

and

$$
A_{\xi-\eta}^{3} X=\left(A_{\xi}^{3}-A_{\xi}^{2} A_{\eta}-A_{\xi} A_{\eta} A_{\xi}+A_{\xi} A_{\eta}^{2}-A_{\eta} A_{\xi}^{2}+A_{\eta} A_{\xi} A_{\eta}+A_{\eta}^{2} A_{\xi}-A_{\eta}^{3}\right) X .
$$

Combining the above equations, we have

$$
\begin{gathered}
\left(\|\xi+\eta\|^{2}-\|\xi-\eta\|^{2}\right) X+\left(\|\xi+\eta\|^{2}+\|\xi-\eta\|^{2}\right)\left(Y_{+}+Y_{-}+Y_{0}\right) \\
=2\left(A_{\xi} A_{\eta} A_{\xi}+A_{\eta} A_{\xi}^{2}+A_{\xi}^{2} A_{\eta}+A_{\eta}^{3}\right) X=8 Y_{+}+4 Y_{-}+4 Y_{0} .
\end{gathered}
$$

Taking $T^{+}$-components and using $\|\xi+\eta\|^{2}-\|\xi-\eta\|^{2}=4 g(\xi, \eta)$, we get $Y_{+}=$ $g(\xi, \eta) X$. If $\nu=g(\xi, \eta)$, then $A_{\eta} X=\nu X+Y_{-}+Y_{0}$. By similar arguments, for $X \in T^{-}$, if we put $A_{\eta} X=Y_{+}+Y_{-}+Y_{0}$, then $Y_{-}=-g(\xi, \eta) X$, and $A_{\eta} X=Y_{+}-\nu X$ $+Y_{0}$, where $\nu=g(\xi, \eta)$. Consequently,

$$
A_{\eta}=\left(\begin{array}{ccc}
\nu I_{m} & { }^{t} B & { }^{t} C \\
B & -\nu I_{m} & { }^{t} D \\
C & D & 0
\end{array}\right),
$$

so that

$$
A_{\xi} A_{\eta}=\left(\begin{array}{ccc}
\nu I_{m} & { }^{t} B & { }^{t} C \\
-B & \nu I_{m} & -{ }^{t} D \\
0 & 0 & 0
\end{array}\right),
$$

$0=\langle\xi, \eta\rangle=\operatorname{trace} A_{\xi} A_{\eta}=2 m \nu$, and $\nu=0$.

Since the matrix of the almost complex structure $J$ is

$$
J=\left(\begin{array}{ccc}
0 & -I_{m} & 0 \\
I_{m} & 0 & 0 \\
0 & 0 & J^{\prime}
\end{array}\right),
$$


using the fact that $N$ is a Kähler submanifold, we obtain

$$
A_{J \eta}=J A_{\eta}=\left(\begin{array}{ccc}
-B & 0 & -{ }^{t} D \\
0 & { }^{t} B & { }^{t} C \\
J^{\prime} C & J^{\prime} D & 0
\end{array}\right) .
$$

Thus $B=0$ because $\langle\xi, J \eta\rangle=0$. By putting $C_{\eta}=C$, we complete the proof.

We choose a local field $\xi_{1}, \ldots, \xi_{p}, J \xi_{1}=\xi_{1}^{*}, \ldots, J \xi_{p}=\xi_{p}^{*}$ of orthonormal frames of $T^{\perp}(N)$. Let $\sigma$ be the second fundamental form, $\nabla^{\prime}$ the covariant differentiation with respect to the connection in (tangent bundle) $\oplus$ (normal bundle), and $\Delta$ the Laplacian.

Proposition 4.2 [10]. Let $M^{n+p}(c)$ be an $(n+p)$-dimensional complex space form of constant holomorphic sectional curvature $c$, and let $N$ be an $n$-dimensional Kähler submanifold of $M$. Then

$$
\begin{aligned}
(1 / 2) \Delta\|\sigma\|^{2}= & \left\|\nabla^{\prime} \sigma\right\|^{2}-2 \operatorname{trace}\left(\sum_{\alpha} A_{\alpha}^{2}\right)^{2}-\sum_{\alpha, \beta}\left(\operatorname{trace} A_{\alpha} A_{\beta}\right)^{2} \\
& +c(n+2)\|\sigma\|^{2} / 2, \text { where } \alpha, \beta=1, \ldots, p, 1^{*}, \ldots, p^{*} .
\end{aligned}
$$

5. Proof of theorems. We prove Theorems 2 and 3. By assumption, $\|\sigma\|^{2}$ is constant so that $\Delta\|\sigma\|^{2}=0$. We compute the right-hand side of (4.2). Since $\operatorname{trace} A_{\alpha}^{2}=2 m,\|\sigma\|^{2}=\sum \operatorname{trace} A_{\alpha}^{2}=4 m p, \quad$ and $\sum\left(\operatorname{trace} A_{\alpha} A_{\beta}\right)^{2}=\sum\left(\operatorname{trace} A_{\alpha}^{2}\right)^{2}=$ $8 m^{2} p$, where $A_{\alpha}$ stands for $A_{\xi_{a}}$. Next, $\left(\sum A_{\alpha}^{2}\right)^{2}=2 \sum A_{\alpha}^{4}+\sum_{\alpha \neq \beta, \beta^{*}} A_{\alpha}^{2} A_{\beta}^{2}$. We have $\operatorname{trace} \sum A_{\alpha}^{4}=4 m p$. Using (4.1), we get trace $A_{\xi}^{2} A_{\eta}^{2}=2$ trace ${ }^{\prime} C_{\eta} \cdot C_{\eta}$. Since $2 m=$ trace $A_{\eta}^{2}=4$ trace ${ }^{t} C_{\eta} \cdot C_{\eta}$, we obtain trace $A_{\xi}^{2} A_{\eta}^{2}=m$. Hence trace $\sum_{\alpha \neq \beta, \beta^{*}} A_{\alpha}^{2} A_{\beta}^{2}=$ $4 m p(p-1)$. Since $c=4$, (4.2) implies

$$
\left\|\nabla^{\prime} \boldsymbol{\sigma}\right\|^{2}=8 m p(p+m-n-1) .
$$

On the other hand, from Remark 3.5, using the notions of $\S 3$, we get $m=m_{2}$, $2 p=m_{1}+2$ and $2 n=m_{1}+2 m_{2}$, so that $p+m-n-1=0$. Consequently, $N$ is a parallel Kähler submanifold in $P^{n+p}(\mathbf{C})$. Parallel Kähler submanifolds of a complex projective space have been completely classified by Nakagawa and Takagi [8] as $P^{n}, Q^{n}, P^{k} \times P^{n-k}, S U(n+2) / S(U(2) \times U(n))(n \geqslant 3), S O(10) / U(5)$ and $E_{6} / \operatorname{Spin}(10) \times T^{1}$.

All principal curvatures of a totally geodesic submanifold are zero, and $Q^{n}$ has two distinct principal curvatures for any normal directions [13]. Moreover, it is known by Ogiue [10] that principal curvatures of Veronese imbedding of the $n$-dimensional complex projective space of constant holomorphic sectional curvature 2 into $P^{n(n+3) / 2}$ depends on the normal direction, hence it does not satisfy the conditions of Theorem 3. Consequently, we check the remaining examples.

Case 1. $P^{k} \times P^{n-k} \rightarrow P^{n k-k^{2}+n}$. In this case, since $p=n k-k^{2}$, the Gauss equation yields $\|\sigma\|^{2}=8 k(n-k)$. If this submanifold satisfies the conditions of Theorem 3, then $\|\sigma\|^{2}=4 m p=4 m k(n-k)$, so that $m=2$. Moreover, since $n=p+m-1=n k-k^{2}+1$, we get $k=1$ or $n-1$. 
Case 2. $S U(k+2) / S(U(k) \times U(2)) \rightarrow P^{\left(k_{k}{ }^{2}\right)-1}(k \geqslant 3)$. In this case, $n=2 k$, $p=\left(k^{2}-k\right) / 2$ and from the Gauss equation and Table 2 in [8], $\|\sigma\|^{2}=8 k(k-1)$. If the conditions of Theorem 3 are satisfied $\|\sigma\|^{2}=4 m p=2 m k(k-1)$, so that $m=4$. From $n=p+m-1$, we get $2 k=k(k-1) / 2+3$ and hence $k=2$ or 3 . Since $k \geqslant 3$, we have $k=3$.

Case 3. $S O(10) / U(5) \rightarrow P^{15}$. In this case, $n=10, p=5$, and similarly, $\|\sigma\|^{2}=$ 120. If the conditions of Theorem 3 are satisfied, then $\|\sigma\|^{2}=4 p m=20 m$, hence $m=6$ so that $p+m-n-1=0$.

Case 4. $E_{6} / \operatorname{Spin}(10) \times T^{1} \rightarrow P^{26}$. In this case, $n=16, p=10$ and $\|\sigma\|^{2}=320$. If the conditions of Theorem 3 are satisfied, then $\|\sigma\|^{2}=4 p m=40 m$, so that $m=8$. But $p+m-n-1 \neq 0$.

Consequently, this submanifold does not satisfy the conditions of Theorem 3 . Hence the Kähler focal manifold of a real hypersurface of a complex projective space which satisfies the conditions of Theorem 2 is $P^{1} \times P^{n-1}$ or $S U(5) / S(U(3) \times U(2))$ or $S O(10) / U(5)$. On the other hand, since homogeneous real hypersurfaces in a complex projective space with $d=5$ satisfy the assumptions of Theorem 2 , their Kähler focal manifolds must be one of the above examples. More precisely, using the Table of [17], we see that the focal manifold of type $C$ is $P^{1} \times P^{n-1}$, the focal manifold of type $D$ is $S U(5) / S(U(3) \times U(2))$ and the focal manifold of type $E$ is $S O(10) / U(5)$, where types $C, D$ and $E$ mean hypersurfaces which are orbits of the isotropy representation of compact Hermitian symmetric spaces of rank 2 of types $C, D$ and $E$ respectively. Hence $M$ must coincide with one of the homogeneous examples with $d=5$. This completes the proof of Theorems 2 and 3 .

Combining Propositions 3.3 and 3.4 and Theorem 2, we obtain the following.

THEOREM 4. Let $M$ be a connected real hypersurface in $P^{n}(\mathbf{C})$. Then $M$ has constant principal curvatures and $J \xi$ is principal if and only if $M$ is congruent to an open subset of a homogeneous real hypersurface.

Concerning Kähler submanifolds, using a Theorem of Cecil [3] and Theorem 3, we have

THEOREM 5. Let $N$ be a Kähler submanifold of a complex projective space. Then eigenvalues of the shape operator $A_{\xi}$ are constant in the sense that they depend neither on the point $x$ in $N$ nor on the normal vector $\xi$ if and only if $N$ is congruent to an open subset of $P^{n}$ (totally geodesic), $Q^{n}, P^{1} \times P^{n-1}$ or $S U(5) / S(U(3) \times U(2))$ or $S O(10) / U(5)$.

REMARK. The above examples are only "normally homogeneous complex submanifolds in $P^{n}(\mathrm{C})$ " [2].

6. Characterization of noncomplex focal submanifolds of homogeneous real hypersurfaces in $P^{n}(\mathrm{C})$.

THEOREM 6. Let $M$ be a connected real submanifold in $P^{n}(\mathbf{C})$ with codim $\operatorname{R}_{\mathbf{R}} M \geqslant 2$. Suppose $J T^{\perp}(M) \subset T(M)$, and $J \xi$ is an eigenvector of $A_{\xi}$ for any $\xi \in T^{\perp}(M)$. If the principal curvatures of $M$ are constant, that is, eigenvalues of the shape operators 
depend neither on a point of $M$ nor on the normal vector, then $M$ is congruent to an open subset of the noncomplex focal variety of a homogeneous real hypersurface in $P^{\prime \prime}(\mathbf{C})$, and $M$ is minimal.

Proof. Let $N=\phi_{r}(M)$ be a tube of radius $r$ over $M$ for sufficiently small $r>0$. From our assumption and Proposition 2.4, $N$ has constant principal curvatures and $J \xi$ is a principal vector, where $\xi$ is a unit normal vector to $N$. Then Theorem 4 implies that $N$ is an open subset of a homogeneous real hypersurface in $P^{n}(\mathbf{C})$. Consequently $M$ is an open subset of a noncomplex focal variety of a homogeneous real hypersurface in $P^{n}(\mathbf{C})$.

Let $\pi: S^{2 n+1} \rightarrow P^{n}(\mathbf{C})$ be the Hopf fibration. Since $\pi^{-1}(M)$ is a focal variety of an isoparametric hypersurface in $S^{2 n+1}[18], \pi^{-1}(M)$ is a minimal submanifold in $S^{2 n+1}$ [9]. Hence $M$ is a minimal submanifold in $P^{n}(\mathbf{C})$ [11]. This completes the proof.

$M$ is classified in the chart below.

\begin{tabular}{c|c|c|c} 
& $\operatorname{dim}_{\mathbf{R}} M$ & $\operatorname{codim}_{\mathbf{R}} M$ & complex focal variety of $M$ \\
\hline$M_{1}$ & $n(n \geqslant 2)$ & $n$ & $Q^{n-1}$ \\
$M_{2}$ & $4 n-1(n \geqslant 2)$ & 3 & $P^{1} \times P^{n}$ \\
$M_{3}$ & 13 & 5 & $S U(5) / S(U(2) \times U(3))$ \\
$M_{4}$ & 23 & 7 & $S O(10) / U(5)$
\end{tabular}

Remark. Since $M_{j}(j=1,2,3,4)$ is a focal variety of a homogeneous hypersurface, $M_{j}$ is homogeneous. From Theorem 3 is [3], $M_{1}=P^{n}(\mathbf{R})$ (totally geodesic), and using pp. 24-27 in [12], we can see that $M_{2}=\pi(U(n+1) / U(n-1))$ and $M_{3}=\pi(U(5) / \mathrm{Sp}(2) \times U(1))$.

A submanifold $M$ in $P^{n}(\mathbf{C})$ is called totally real if $J T(M) \perp T(M)$.

COROllary. Let $M$ be an n-dimensional totally real submanifold in $P^{n}(\mathbf{C})$. Suppose $J \xi$ is an eigenvector of $A_{\xi}$ and the eigenvalues of $A_{\xi}$ are constant for any $\xi \in T^{\perp}(M)$. Then $M$ is a totally geodesic $P^{n}(\mathbf{R})$.

\section{BIBLIOGRAPHY}

1. U. Abresch, Isoparametric hypersurfaces with four or six distinct principal curvatures, Math. Ann. 264 (1983), 283-302.

2. M. Buchner, K. Fritzsche and T. Sakai, Geometry and cohomology of certain domains in the complex projective space, J. Reine Angew. Math. 323 (1981), 1-52.

3. T. Cecil, Geometric applictions of critical point theory to submanifolds of complex projective space, Nagoya Math. J. 55 (1974), 5-31.

4. T. Cecil and P. Ryan, Focal sets and real hypersurfaces in complex projective space, Trans. Amer. Math. Soc. 269 (1982), 481-498.

5. Y. Macda. On real hypersurfaces of a complex projective space. J. Math. Soc. Japan 28 (1976), 529-54().

6. H. Münzner, Isoparametrische Hyperflächen in Sphären. I, Math. Ann. 251 (1980), 57-71.

7. Isoparametrische Hyperflächen in Sphären. II, Math. Ann. 256 (1981), 215-232.

8. H. Nakagawa and R. Takagi, On locally symmetric Kaehler submanifolds in a complex projective space. J. Math. Soc. Japan 28 (1976), 638-667. 
9. K. Nomizu, Elie Cartan's work on isoparametric families of hypersurfaces, Proc. Sympos. Pure Math., vol. 27, Part I, Amer. Math. Soc., Providence, R. I., 1974, pp. 191-200.

10. K. Ogiue, Differential geometry of Kaehler submanifolds, Adv. in Math. 13 (1974), 73-114.

11. M. Okumura, Submanifolds of real codimension of a complex projective space, Atti Accad. Naz. Lincei 58 (1975), 544-555.

12. H. Ozeki and M. Takeuchi, On some types of isoparametric hypersurfaces in spheres. II, Tôhoku Math. J. 28 (1976), 7-55.

13. B. Smyth, Differential geometry of complex hypersurfaces, Ann. of Math. (2) 85 (1967), 246-266.

14. H. Takagi, A condition for isoparametric hypersurfaces of $S^{\prime \prime}$ to be homogeneous, Tôhoku Math. J. 37 (1985), 241-250.

15. R. Takagi, On homogeneous real hypersurfaces in a complex projective space, Osaka J. Math. 10 (1973), 495-506.

16. _ Real hypersurfaces in a complex projective space with constant principal curvatures, J. Math. Soc. Japan 27 (1975), 43-53.

17. __ Real hypersurfaces in a complex projective space with constant principal curvatures. II, J. Math. Soc. Japan 27 (1975), 506-516.

18. Q. Wang. Isoparametric hypersurfaces in complex projective spaces, Proc. 1980 Beijing Sympos. on Differential Geometry and Differential Equations, Science Press, Beijing, Gordon and Breach, New York, 1982. pp. 1509-1523.

Department of Mathematics, Tokyo Metrpolitan University, Fukazawa, Setagayaku, Tokyo 158, JAPAN 